\title{
CANCER BIOLOGY DATA CURATION AT THE MOUSE TUMOR BIOLOGY DATABASE (MTB)
}

\section{Debra M. Krupke, Dale A. Begley, Steven B. Neuhauser, Joel E. Richardson, John P. Sundberg, Carol J. Bult, and Janan T. Eppis.}

\section{MTB}

Mouse Tumor Biology Database

ABSTRACT

Many advances in the field of cancer biology have been Biology (MTB, http://tumor.informatics.jax.org) database provides web-based access to data on spontaneous and induced tumors from genetically defined mice (inbred, hybrid, mutant, and genetically engineered strains of mice). These data include standardized tumor names and classifications, pathology reports and images, mouse genetics, genomic and cytogenetic changes occurring in the tumor, strain
tency, and literature citations.

Although primary source for the data represented in MTB is peerhistopathology images and cytogenetic assay images for mouse tumors where these data are available from The Jackson Laboratory's mouse colonies and from outside contributors. MTB from the cancer research community and provides investigators web-accessible tool for image submission and annotation Integrated searches of tor use of several controlled vocabularies and by adherence to standard nomenclature. MTB also provides links to other related
online resources such as the Mouse Genome Database, Mouse Phenome Database, the Biology of the Mammary Gland Web Site, Festing's Listing of Inbred Strains of Mice, the JAX Consortium's Mouse Repository.

MTB provides access to data on mouse models of cancer in the internet and has been designed to facilitate the selection of experimental models for cancer research, the evaluation of of mutatiotic models of human cancer, the review of patterns that are commonly mutated across a spectrum of
MTB is supported by

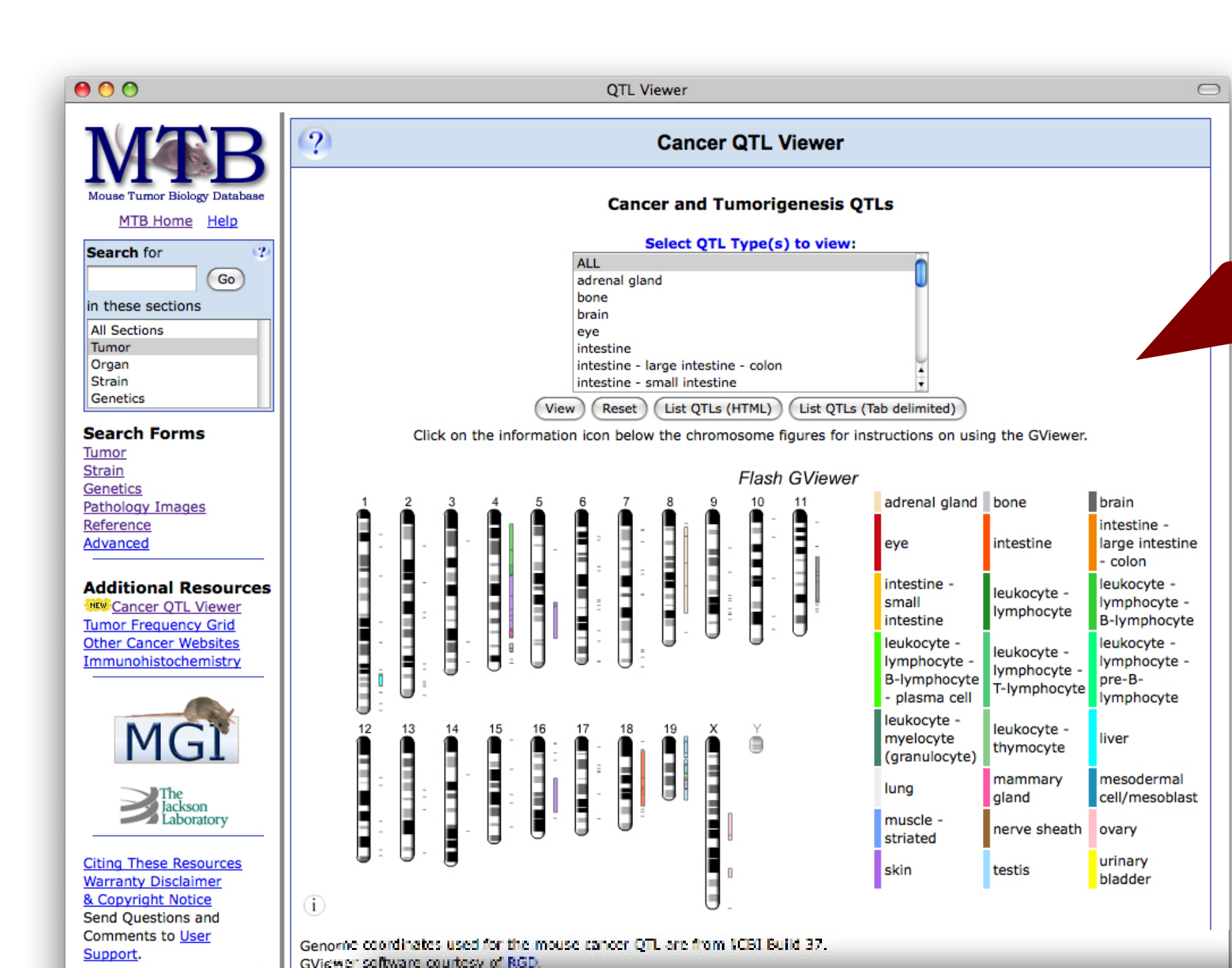

Cancer QTL data are imported from MGD and displayed using GViewer.

\section{Database Stats}

33,732 tumor frequencies

3,594 strains

3,333 pathology images

169 cytogenetic images
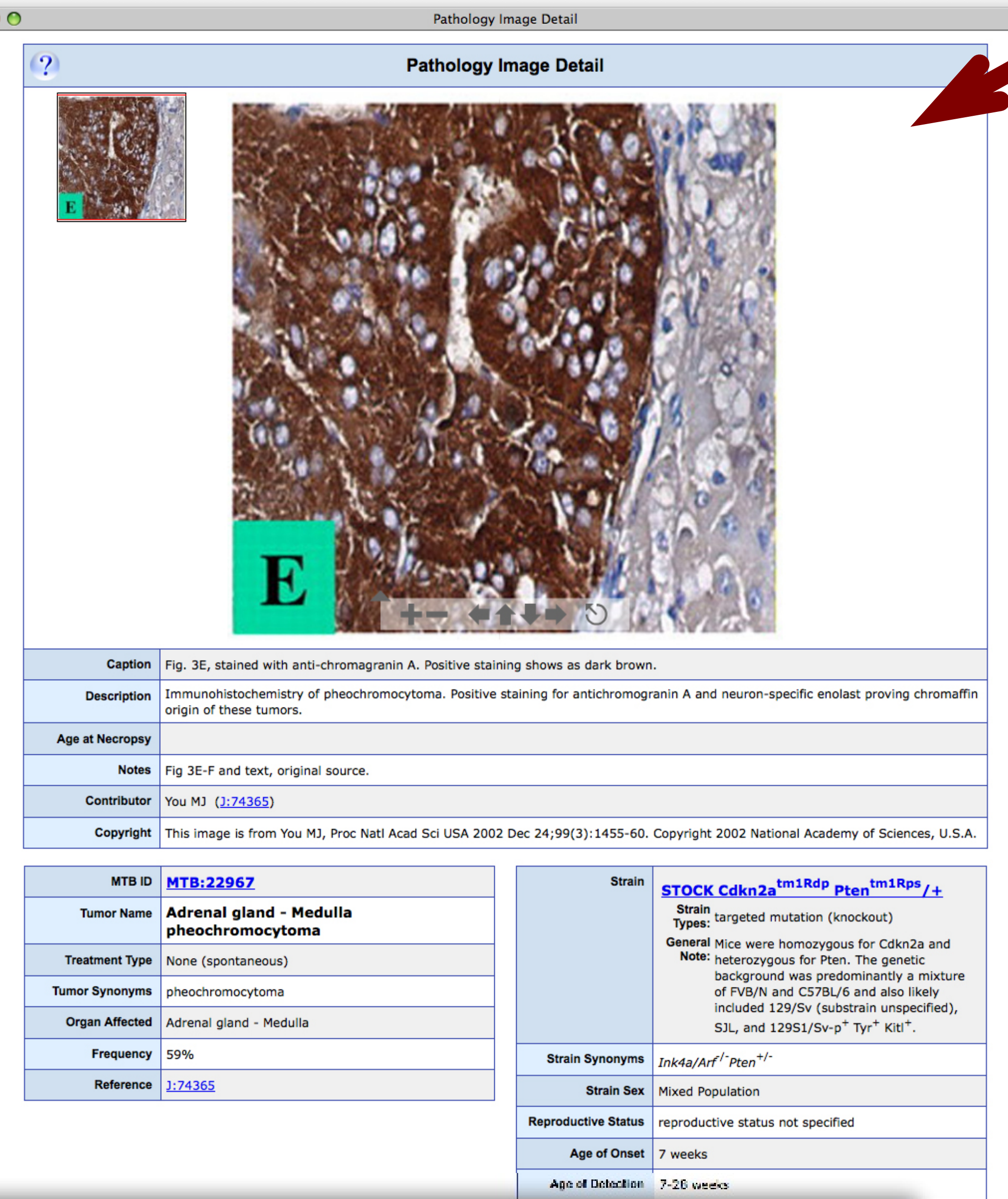

\section{http://tumor.informatics.jax.org}

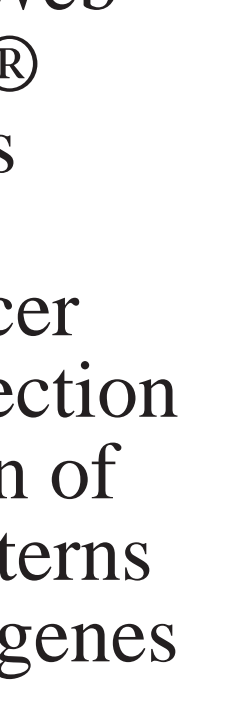
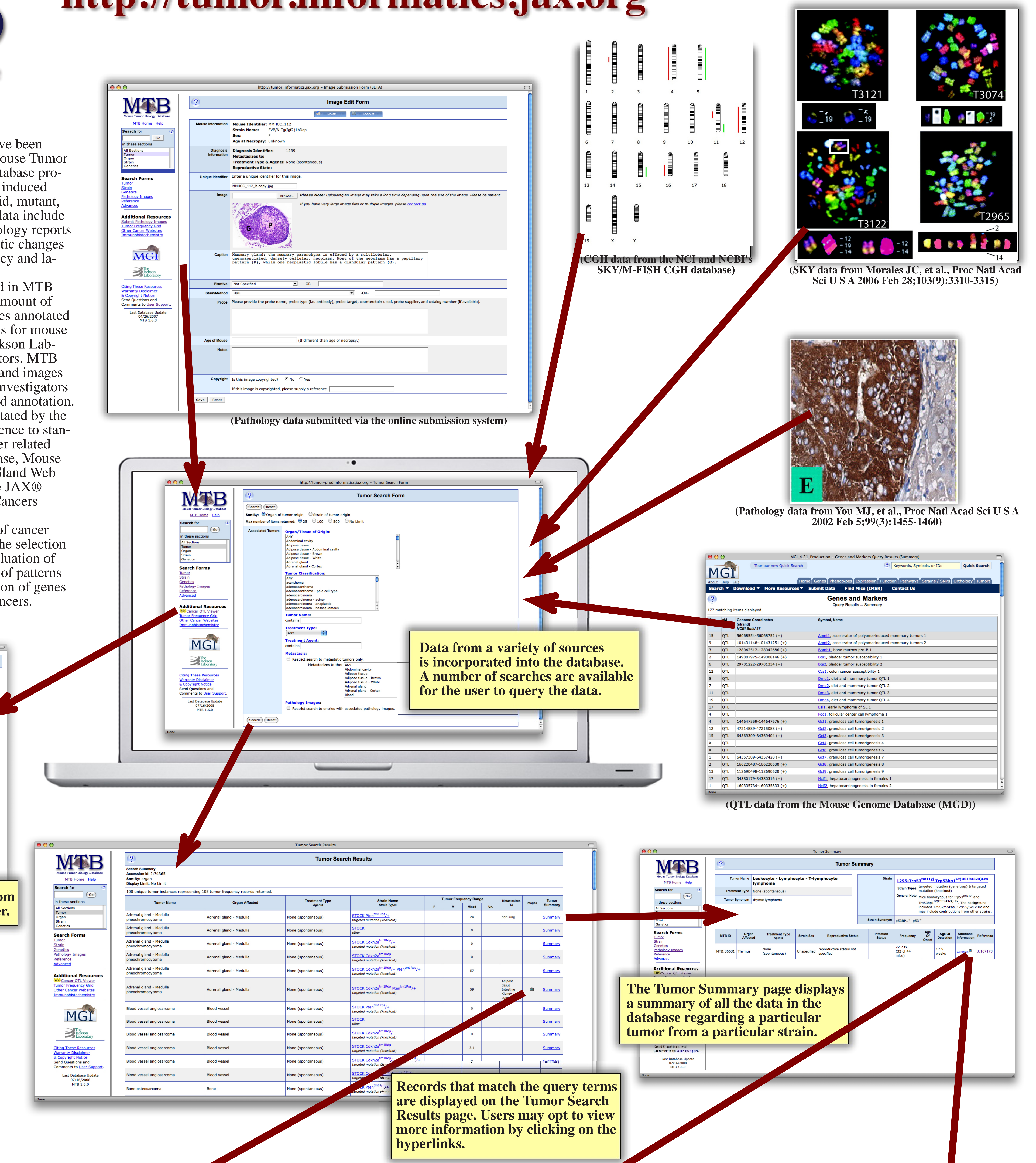